\title{
Unbiased Geometry Optimisation of Morse Atomic Clusters
}

\author{
Wayne Pullan
}

\begin{abstract}
This paper presents the results obtained using an unbiased Population Based Search (PBS) for optimising Morse atomic clusters. PBS is able to repeatedly obtain all putative global minima for Morse clusters in the range $5 \leq N \leq$ $80, N=147, \rho=3,6,10,14$, as reported in the Cambridge Cluster Database. In addition, putative global minima have been established for Morse clusters in the range $81 \leq N \leq$ $146, \rho=14$. The PBS algorithm incorporates and extends key techniques that have been developed in other cluster optimisation algorithms over the last decade. Of particular importance are the use of cut and paste operators, structure niching and a new operator, Directed Optimisation, which extends the previous concept of directed mutation. In addition, PBS is able to operate in a parallel mode for optimising larger clusters.
\end{abstract}

Keywords: global geometry optimisation, clusters, Lennard-Jones, Morse.

\section{INTRODUCTION}

The goal of structural optimisation of atomic clusters, where each atom interacts with all other atoms through a twobody central force, is to identify the structure corresponding to the global minimum potential energy for the cluster. To date, the most commonly studied cluster optimisation problem has been the Lennard-Jones (LJ) cluster where the potential energy arising from the interaction of 2 atoms separated by distance $r$ is given by:

$$
v(r)=4 \epsilon\left(\left(\frac{\sigma}{r}\right)^{12}-\left(\frac{\sigma}{r}\right)^{6}\right)
$$

where $\epsilon=\sigma=1$ (Figure 1). The total energy $E$ of a cluster of $N$ atoms is simply given by the sum of the pair interactions between all atom pairs, i.e.

$$
E(X)=E\left(X_{1}, \ldots, X_{N}\right)=\sum_{i<j} v\left(\left\|X_{i}-X_{j}\right\|\right),
$$

where $X_{i} \in \mathbb{R}^{3}$ are the coordinates of the centre of the $i$-th atom and the norm is the Euclidean one.

The minimisation problem is the following:

$$
\min _{X \in \mathbb{R}^{3 n}} E(X) .
$$

It is important to remark here that we are interested in the global minimum for this problem as local minima for this problem can be detected quite efficiently but it is conjectured that their number increases exponentially with the number $N$ of atoms. This makes the global minimisation problem a difficult one which has been extensively studied and has many applications. For example, it is employed in the analysis of the three-dimensional conformation of clusters of

Wayne Pullan is with the School of Information and Communication Technology, Griffith University, Gold Coast, Qld., 4215, Australia. ( Email: w.pullan@griffith.edu.au) inert atoms. Moreover, although the Lennard-Jones potential is not a good choice when modelling metals, in some cases (e.g. gold or nickel) there is a strong preference for the formation of 75-atom clusters with the same structure as the optimal Lennard-Jones cluster of that size. In addition, the Lennard-Jones pair-potential is an important component of the non-bonded pair interactions in complex molecular systems such as proteins. In studying the three-dimensional conformation of proteins it is recognised that this interaction, together with the electrostatic, or Coulomb, interaction, is the most relevant one. Therefore, developing efficient methods for the minimisation of the Lennard-Jones clusters could be an important step towards developing efficient methods for the protein folding problem.

As pointed out in [20], the optimal structures for LJ clusters are quite structurally uniform with virtually all of the global minima structures based on the Mackay icosahedra. Considerably more difficult optimisation problems are the optimisation of atomic clusters where the potential energy arising from the interaction of 2 atoms is given by the following Morse potential:

$$
v_{\rho}(r)=\left(e^{\rho(1-r)}-1\right)^{2}-1
$$

The form of $v_{\rho}(r)$ is shown in Figure 1 where the minimum of $v_{\rho}(r)$, for all values of $\rho$, occurs at $r=1$. The single

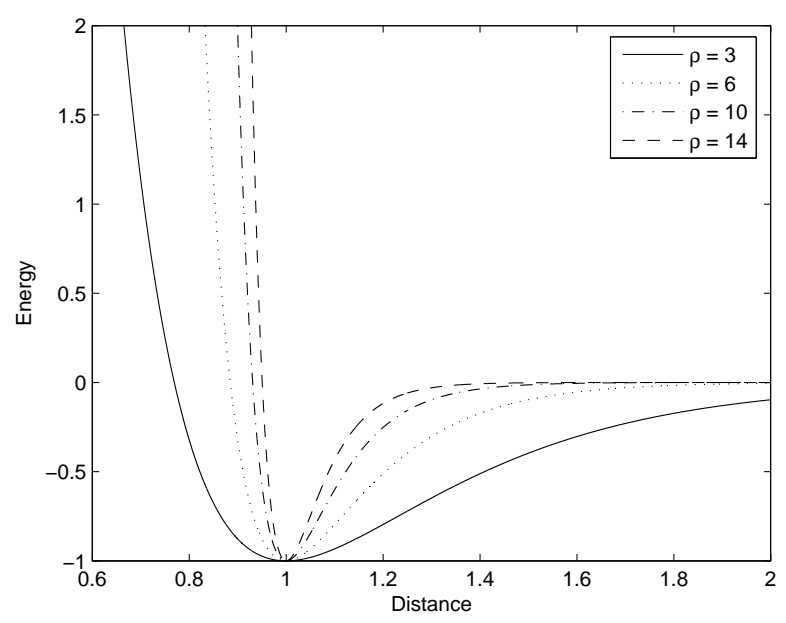

Fig. 1. Morse $(\rho=3,6,10,14)$ pair-potentials.

parameter $\rho$ for the Morse potential directly controls the range of the potential (Figure 1) and allows a wide range of interactions to be modelled. When $\rho=6$, the Morse potential is close to the LJ potential and gives rise to similar optimal icosahedral structures. When $\rho<6$ the potential energy 
hyper-surface for the Morse cluster becomes smoother and optimal structures tend to be polytetrahedral. As $\rho$ increases from 6 to 14, the potential energy hyper-surface becomes more rugged, with larger barriers between an increasing number of local minima, and optimal structures transition from icosahedral to decahedral to close-packed [20].

This paper describes the application of an unbiased population based optimisation method, PBS [18] to Morse clusters and is structured as follows: in Section II, an overview of previous LJ and Morse cluster optimisation methods is presented, particularly those relevant to the PBS algorithm described in Section III. The results achieved by PBS for Morse clusters are shown in Section IV while Section V contains a conclusion and possible future research directions.

\section{HistoricAl OVERVIEW}

\section{A. Lennard-Jones Clusters}

Prior to 1987 , the most extensive study of Lennard-Jones clusters was the work of Hoare et al. [1], [4], [5], [6] who developed a general growth algorithm and used it to generate large numbers of stable structures, mainly for $N \leq 55$. These were compared to find the lowest energy structures which, in turn, became candidates for the absolute minimal structures. Hoare and Pal [5] observed that, while what they termed as the "icosahedral growth sequence" did not, in general, produce minimal structures, icosahedral subunits did appear regularly in relaxed configurations generated by other sequences. The icosahedral lattice can be described as 20 slightly flattened tetrahedrally shaped face-centred-cubic units with 12 vertices on a sphere centred at the origin. For the icosahedral lattice, the total number of lattice points on each layer is $1,12,42,92,162,252, \ldots$ Therefore, the number of lattice points in the sequence of closed shell icosahedral lattices is $1,13,55,147,309,561, \ldots$.

The observation by Hoare and Pal led to the so-called lattice search methods, where the search for optimal clusters was performed over an icosahedral lattice [7], [8]. Using these techniques, many putative LJ global minima for $N \leq 147$ [7] were discovered for the first time. Subsequently, lattice search methods have produced the putative global minima for $148 \leq N \leq 309$ [9], $310 \leq N \leq 561$ [10] and $562 \leq N \leq 1000$ [11]. However, as lattice based search methods are biased (i.e. the search is restricted to only a portion of the feasible domain, namely that portion containing icosahedral clusters), they do not explore other regions which, in some cases, contain lower energy clusters with a basically non-icosahedral structure.

Over the last decade a number of optimisation methods have evolved for the unbiased, systematic optimisation of LJ clusters. These can be broadly grouped into Non-Population and Population based categories and are now discussed in more detail.

Unbiased Non-Population based search - With regard to unbiased, non-population based search methods, one of the most powerful is the Basin-Hopping method [12] which exploits the funnel structure of the energy landscape. A funnel can be defined as a set of local minima such that, for all of them, at least one decreasing sequence of "neighbour" local minima exists leading to a unique local minimum corresponding to the bottom of the funnel. The key observation was that, while LJ clusters have a large number of local energy minima (conjectured to be exponentially increasing with the number $N$ of atoms), the number of funnels is usually very limited (in some easy cases, such as $N=13$, there even exists a single funnel) and, for one of these funnels, the bottom is always the global minimum of the energy function. This observation led to the introduction of the Basin-Hopping $(\mathrm{BH})$ algorithm [12] which is able to reach the bottom of a funnel and was the first unbiased algorithm to detect all putative global minima, as currently reported in [2], up to $N=110$ atoms. $\mathrm{BH}$ randomly explores local minima returned by local searches started within a neighbourhood of the current local minimum until it decides, according to a Metropolis acceptance criterion, to move to one of those local minima or to stop because a prefixed number of iterations with no improvement has been reached. Since there is no guarantee that a single run of $\mathrm{BH}$ is able to detect the global minimum (at least within a reasonable amount of time), $\mathrm{BH}$ is usually employed in a multi-start fashion, i.e. it is run many times from different, randomly sampled, starting points. In spite of its simplicity, the successes of $\mathrm{BH}$ were impressive and its monotonic variant $\mathrm{MBH}$ [13] was able to detect a new optimal structure for the case of $N=98$ atoms, the Leary tetrahedron [14].

Recently the $\mathrm{BH}$ method has been extended by the incorporation of Two-phase local searches [15]. In these local searches, the local minimisation of the original energy function $E$ is preceded by the local minimisation of a modified function $F=E+g$ where function $g$ is a parameterised geometric penalisation term which allows different geometrical shapes to be favoured. In principle, local searches could be substituted by two-phase local searches in all algorithms. When incorporated into $\mathrm{MBH}$, two-phase local searches considerably improve the efficiency of detecting the most challenging LJ global minima, namely those having a non-icosahedral structure (the FCC structure at $N=38$, the decahedral structure at $N=75-77,102-104$, and the already mentioned Leary tetrahedron at $N=98$ ).

Unbiased Population based search - In population based approaches, for example [16], [17], efficiency is increased by keeping, at each iteration, not a single local minimum as in BH but a population of local minima in such a way that all of them "sufficiently" differ from each other. Basically, diversification is forced between members of the population and many trajectories are followed at the same time which often improves the performance with respect to methods, such as BH, which only follow a single trajectory 
at a time and can only force diversification through random restarting.

Over the last decade, a number of successful, unbiased, population based, search methods have evolved for the systematic optimisation of LJ clusters. These include the genetic algorithm of Deaven et al. [16] which introduced the concept of phenotype cut and paste operators for crossover and mutation. This algorithm was able to find most putative global minima up to $N=110$ but failed for some cluster sizes where the optimal structure is non-icosahedral (the Face Centred Cubic (FCC) structure at $N=38$, the decahedral structures at $N=75-77$, the Leary tetrahedron at $N=98$ and the decahedral structures at $N=102-104$ ). Subsequently, Hartke [17] developed a phenotype population based algorithm introducing the concepts of structure niching and directed mutation. This algorithm was able to find, with the exception of $N=98$, all putative global minima in the range $2 \leq N \leq 150$.

A more recent unbiased population based approach, PBS, combines and extends phenotype operators, structure niching and directed mutation and two-phase local searches into a parallel processing environment and was able to find all putative global minima in the range $2 \leq N \leq 372$ [18]. Within the context of unbiased population based search, the techniques of cut and paste operators, structure niching and directed mutation seem to be keys to the improved performance of these algorithms and they are now discussed in more detail:

- Cut and Paste (Phenotype) Operators In BH the generation of a new local minimum is simply obtained by starting a local search within the neighbourhood of the current local minimum. In the framework of population based approaches, a number of new operators have been defined that generate a new cluster by modifying a cluster (unary operators) or by combining two clusters (binary operators). As these operators function directly on the geometric model of the cluster, they have a greater probability of retaining good "building blocks" within the cluster when compared to operators that function on the genotype representation of the cluster.

- Structure Niching is a population diversification technique implemented through dissimilarity metrics, which measure either the relative difference between clusters, or produce an absolute measure of cluster structure [17]. These structure metrics allow structure niche groups to be maintained within the population. During population updating, the energy value of a cluster to be added to the population is only compared with the energy values of the clusters within the same structure group. This ensures that non-icosahedral structures are not eliminated from the population during the search by the more prevalent and, initially lower energy, icosahedral structures.

- Directed Mutation It has been observed that the performance of $\mathrm{BH}$ degrades as the number $N$ of atoms increases ${ }^{1}$. It can be reasonably claimed that one of the main reasons for this lies in the mechanism that generates new candidate local minima in the neighbourhood of the current local minimum. In BH this is simply obtained by a random perturbation of all the coordinates of the current local minimum. This mechanism often leads quickly to a point close to the global minimum and is also quite general (i.e. it can be extended to global optimisation problems that are different from molecular conformation ones). However, it often happens that a better local minimum than the current one exists but is only slightly different from the current one. Such an improvement is difficult to detect by perturbing all the atoms in a cluster (thus disrupting the whole structure of the cluster), and this difficulty increases with the number $N$ of atoms. Therefore, the key to improving the performance is to find other, more structured, perturbations of atoms in addition to using random perturbations. Hartke [17] implemented such a directed mutation technique by moving the "worst" atom to the "best" vacant position and observed that if directed mutation is employed "the resulting overall speedup can be so large that it makes all the difference between an efficient solution and impractically long computation times". In addition, similar angular moves of the most weakly bound surface atoms were implemented in [12] and also within the GMIN computer program [2].

A further inherent benefit of population based algorithms is that they are often straight forward to parallelise with the obvious, but important consequence, of a considerable reduction in elapsed times for optimising clusters.

\section{B. Morse Clusters}

As a function of $\rho$, Morse clusters have a much wider range of structures than Lennard-Jones clusters. In addition, the potential energy landscape also changes with $\rho$, with the number of local minima increasing rapidly changing the landscape from smooth to rough. This makes the global optimisation of Morse clusters for $\rho=10$ and $\rho=14$ a very difficult task and is orders of magnitude more difficult than the optimisation of the corresponding size Lennard-Jones cluster. A database of putative global minima is available for Morse clusters [2].

In contrast to the widely studied Lennard-Jones clusters, very few global optimisation algorithms have been developed for Morse clusters. In [19], a genetic algorithm is described which is successful for small and medium size $(N \leq 50)$ Morse clusters while the potential energy transformation algorithm [20] is successful for all clusters documented in [2]. The PBS algorithm documented in this paper is the first population-based algorithm to be successful for all clusters documented in [2]. in addition, PBS has produced putative minima for Morse clusters in the range $81 \leq N \leq 146$ with $\rho=14$.

\footnotetext{
${ }^{1}$ Private communication
} 


\section{Population Based Search Algorithm}

The Population Based Search (PBS) presented in this paper is tailored to cluster optimisation problems and utilises several of the key techniques described in Section II. PBS is able to efficiently utilise any number of computer processors to optimise clusters. A Master task controls some number of sub-tasks using a simple MPI send-receive message / command interface. The Master task manages the population and allocates work to sub-tasks that either generate a new member for the population, mutate an existing member, or perform a cross-over of two existing members. There is no concept of "generations" and the only task synchronisation point occurs at the end of the population creation phase.

PBS is a two-phase algorithm with an initial population creation phase (III-B) which parallels the Basin-Hopping method in that it randomly generates starting trial clusters and incorporates mechanisms for following the energy funnel within which the cluster lies. The primary goal of the population creation phase is to create an initial population, of eight members, that contains a range of structures, all with energies reasonably close to the global minimum. This population creation phase is followed by a search phase which starts with the initial population and, using cross-over and mutation operators followed by local searches, applied to all members and all possible pairings of members of the population, iteratively updates the population.

The basic techniques utilised by both the population creation and search phases of PBS are detailed in Section III-A, the population creation phase is presented in Section III-B and the search phase described in Section III-C.

\section{A. Basic PBS Techniques}

The population management and search techniques that are common to both the population creation and search phases of PBS are described in the following sub-sections.

1) Population Updating: As each sub-task returns the results of a cluster generation, mutation or cross-over the master task decides if the new cluster should be added to the population or discarded. Basically, a new cluster will be unconditionally added to the population if its structure niche group is below the maximum allowed group size and the energy of the new cluster is not within 0.1 above of an existing member of the structure niche group. If this is not the case then the new cluster will replace the highest energy member of the structure niche group, provided its energy is less than that of the member.

2) Directed Optimisation: The goal of the Directed Optimisation operator is to iteratively identify and repair surface and interior "defects" in clusters. Directed Optimisation functions in one of the following three modes:

1) Surface Repair - moves the atom with the lowest number of Nearest Neighbour (NN) atoms to the best adjacent vacant position near a target atom with the maximum (but less than 12) number of NN atoms. In this context, NN atoms are those whose squared distance from the target atom is in the range $0.81 \ldots 1.54$.
The best adjacent vacant position is obtained by constructing all possible tetrahedron apexes for the triangles formed by all possible combinations of the target atom and its NN atoms. Basically this mode attempts to move the "worst" surface atom to the "best" available position on the surface and is used during the generation of new clusters and whenever an existing population member is mutated.

2) Interior Repair 1 - randomly selects an atom from the atom pair that are closest to each other (provided the separation is less than 1.123) and removes this from the cluster, locally optimises the remaining $N-1$ cluster using LBFGS and then adds the removed atom back onto the cluster using the Surface Repair technique described above. This mode of Directed Optimisation is used during the generation of all new clusters.

3) Interior Repair $\mathbf{2}$ - randomly selects an atom from all atom pairs whose separation is less than 1.123 , locally optimises the remaining $N-1$ cluster using LBFGS and then adds the removed atom back onto the cluster using the Surface Repair technique described above. This mode is used as a local mutation operator for current population members.

The primary motivations for the Directed Optimisation operator are that, from Figure 1, it is clear that:

- every atom should have the maximum possible number of nearest neighbours. The surface repair mode contributes to this by ensuring that all atoms in the outer "shell" are placed adjacent on the surface of the cluster.

- if two atoms are nearest neighbours, then the distance between them should be as close to 1.123 as possible. The interior repair modes contribute to this by identifying atom pairs that are closer than the optimal distance and creating a situation which rectifies this and also causes a reorganisation of the neighbouring atoms.

Figure 2 shows the performance of the Directed Optimisation operator during the optimisation of the $N=104, \rho=14$ cluster. Given that the Directed Optimisation operator is only applied to a cluster that has already been locally optimised using two-phase local search, it is clearly very effective in following the energy funnel and is, probably, the key factor for the performance of PBS.

\section{B. PBS Population Creation Phase}

PBS uses a technique for initially generating the population which is effective in following energy funnels. Initially PBS generates a maximum of 150 trial solutions, randomly generated within a cube whose volume is dependent on the number of atoms. All trial solutions are subsequently locally optimised and then subjected to the Directed Optimisation (III-A.2) operator. Figure 3 shows the absolute difference between the putative global minima and the best obtained by PBS population creation for clusters in the range $5 \leq$ $N \leq 147$. Generally, the PBS population creation phase is able to generate initial populations which either contain the putative global minimum or contain clusters whose energies 


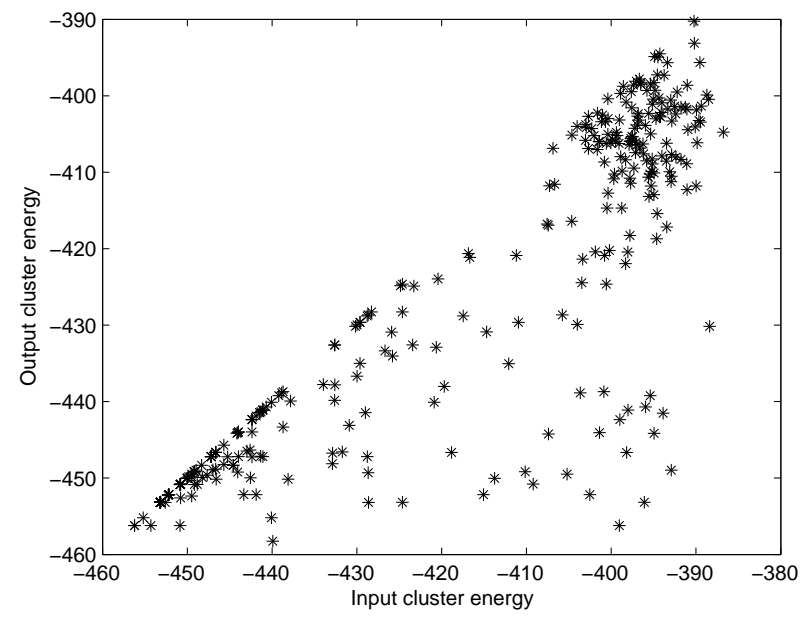

Fig. 2. Energy in versus energy out for the Directed Optimisation operator during the optimisation of an $N=104, \rho=14$ cluster where the putative global energy minimum is -458.252082 . All clusters input to the Directed Optimisation operator have already been locally optimised by the two-phase local search. All points below the diagonal represent an improvement in cluster energy. Note that the Directed Optimisation operator returns the original cluster whenever it is unable to generate an improved cluster.

are reasonably close to the putative global minimum. Clearly this is of considerable benefit to the subsequent PBS search phase.

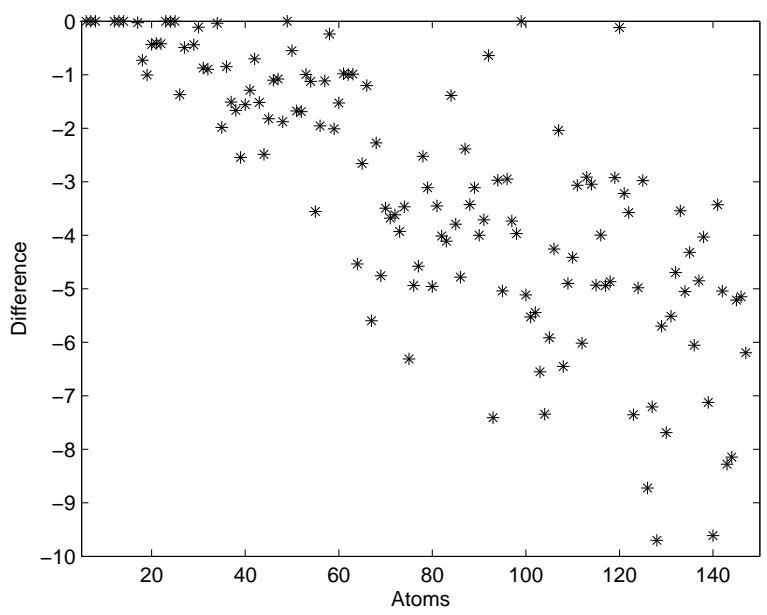

Fig. 3. The difference between the putative global minima and the best energy obtained by PBS population creation phase for $5 \leq N \leq 147$. As can be seen, the population creation phase of PBS is able to find the putative global minima for a considerable range of cluster sizes but becomes less effective as $N$ increases.

\section{PBS Search Phase}

The PBS search phase uses mutation and cross-over operators with a primary goal of generating new starting points for the local optimisation methods described in sub-section III-A.2. The mutation and cross-over operators operate only on population members and function at both large and small

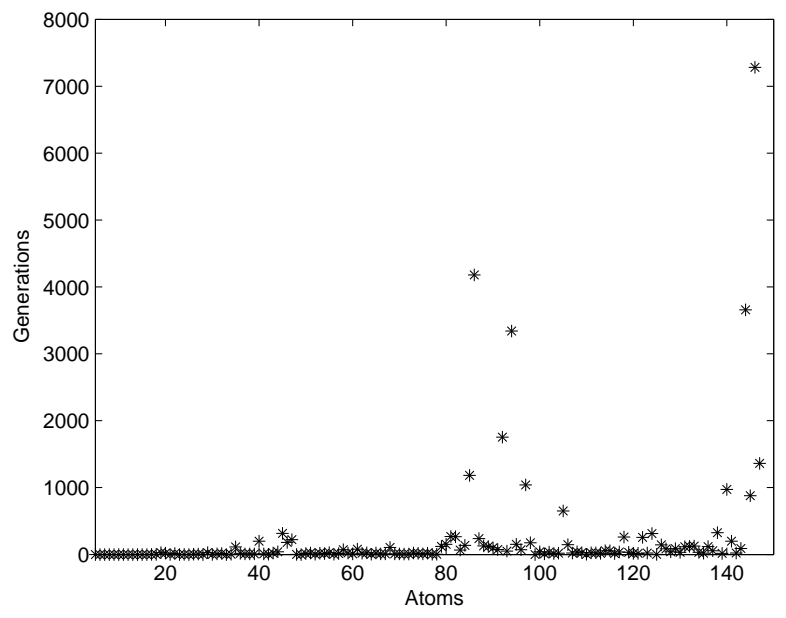

Fig. 4. Number of PBS generations for $5 \leq N \leq 147, \rho=14$.

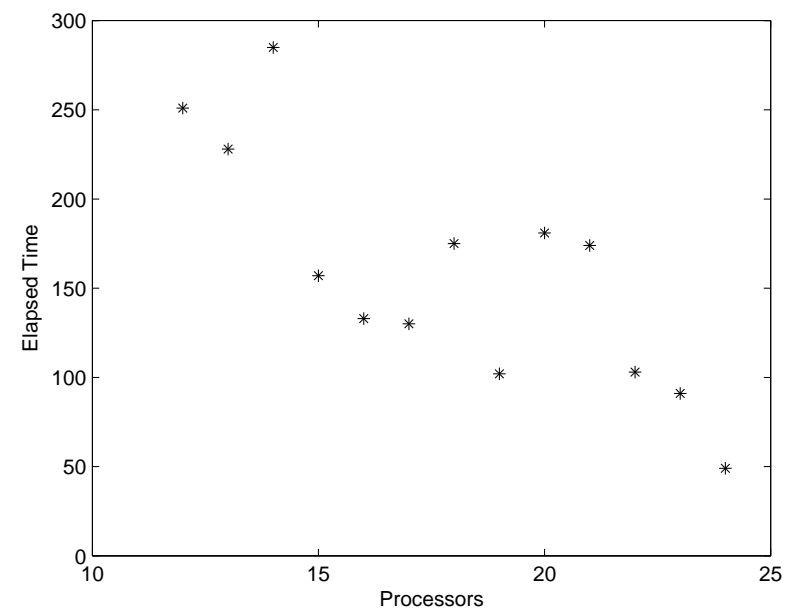

Fig. 5. Processor scaling for $N=72, \rho=14$ cluster.

scales providing both coarse and fine grained search. It should be noted that, at some point during the optimisation of clusters in the range $5 \leq N \leq 147$, all mutation and crossover operators described here were the last operator applied immediately prior to finding at least one global optimum.

1) Coarse-grained Search: The primary role of the crossover and global mutation operators is to move around the search space in large steps. For cross-over, all possible combinations within the population are used and two cross-over operators exist: the random cross-over (used with probability 0.8 ) which randomly rotates the clusters around the three axes, selects some number of atoms and, using the ones most distant from the $x-y$ plane, swaps these by translating atoms using the most distant atom from each cluster as the basis for the translation. The second cross-over operator is the selective cross-over which attempts to combine "good" hemispheres from each cluster when it generates the child clusters. 
The global mutations operate on a single cluster and affect all atoms in the cluster. These are applied to each member of the population and perform twist (atoms are rotated about a random axis, where the angle of rotation increases with distance from the lowest atom on that axis) and perturb (a small, random perturbation of all atoms) operations. In addition, a small number of new clusters are randomly generated during the search phase of PBS.

2) Fine-grained Search: The primary role of the local mutation operators is to move around the search space in small steps. Local mutations are only applied to population members and have a localised effect within a cluster, typically close to a plane through the cluster. The local mutation operators in PBS are slide (cluster segments above and below a random plane are translated parallel to the plane), rotate (cluster segments above and below a random plane are rotated around an axis normal to the plane), self cross-over and the Directed Optimisation operator.

\section{EXPERIMENTAL RESULTS}

The performance metrics used to classify algorithms should be complete in that they measure all of a particular aspect of the algorithm. For example, as a measure of computational effort or run-time, median / average number of local optimisations has been used. However, this measure does not effectively compare algorithms that have different uses for local optimisation (e.g. only optimising near optimal clusters as against optimising random clusters) or where the local optimisations have differing computational overheads. Table I gives performance statistics, from ten trials of the one-processor version of PBS, to successfully optimise all clusters in the range $2 \leq N \leq 80$ with $\rho=14$. The following points are relevant when interpreting the data in Table I.

- For each trial, the maximum allowed generations was set to 100.

- As PBS frequently invokes local optimisations on clusters which are at a local minimum, all local optimisations that required less than 0.0001 seconds of CPU time have not been included in the local optimisation counts.

- The figures for local optimisations and CPU are averaged over successful trials only.

Clearly the use of processor time as a measure of computational effort provides a more encompassing metric as it reflects the total amount of work performed by the search. However, this metric does have the disadvantage that the processor time is clearly dependent on the computer processor used for the test and this makes comparison between algorithms tested on different computers difficult. To overcome this, the method proposed in the COCONUT ${ }^{2}$ project has been used and the computer processor time, in terms of the processor time taken to evaluate the shekel5 function at $1.0 \mathrm{E}+8$ points ${ }^{3}$ is documented. While this provides only a

\footnotetext{
${ }^{2}$ http://www.mat.univie.ac.at/ neum/glopt/coconut

${ }^{3} \mathrm{~A} \mathrm{C}++$ program is available at

http://www.mat.univie.ac.at/ neum/glopt/coconut/shekel5.cpp
}

basic measure of a computer processor, we feel it is adequate for comparing processor time requirements for optimisation algorithms. All one-processor experiments in this paper were performed on a dedicated computer that required 16.69 processor seconds to execute a non-optimised version of shekel5, compiled under Linux using the g++ compiler.

TABLE I

PERFORMANCE STATISTICS FOR THE SINGLE-PROCESSOR VERSION OF PBS OPTIMISING ALL CLUSTERS IN THE RANGE $5 \leq N \leq 80 \mathrm{WITH}$ $\rho=14$. SHOWN ARE THE NUMBER OF ATOMS IN THE CLUSTER, THE

PUTATIVE GLOBAL MINIMUM ENERGY [2] AND THE AVERAGE CPU TIME (SECONDS).

\begin{tabular}{r|r|r|r|c|r|r|r|r}
\hline \hline $\mathrm{N}$ & \multicolumn{1}{c|}{ Opt. } & \multicolumn{1}{|c|}{ CPU } & N & \multicolumn{1}{c}{ Opt. } & \multicolumn{1}{|c|}{ CPU } & N & \multicolumn{1}{c|}{ Opt. } & \multicolumn{1}{c}{ CPU } \\
\hline 5 & -9.000283 & 0.000 & 31 & -111.760670 & 60.430 & 56 & -225.655136 & 309.933 \\
6 & -12.018170 & 0.027 & 32 & -115.767561 & 116.445 & 57 & -230.663986 & 818.621 \\
7 & -15.883113 & 0.000 & 33 & -120.741345 & 40.064 & 58 & -234.809078 & 130.967 \\
8 & -18.883688 & 0.001 & 34 & -124.748271 & 65.941 & 59 & -240.572493 & 493.232 \\
9 & -22.644892 & 0.001 & 35 & -129.737360 & 152.892 & 60 & -244.579066 & 534.762 \\
10 & -26.132735 & 0.003 & 36 & -133.744666 & 185.252 & 61 & -249.587740 & 1946.203 \\
11 & -29.596054 & 0.006 & 37 & -138.708582 & 109.905 & 62 & -253.612942 & 406.549 \\
12 & -33.332305 & 0.103 & 38 & -144.321054 & 157.788 & 63 & -258.620607 & 697.751 \\
13 & -37.258877 & 0.020 & 39 & -148.327400 & 556.861 & 64 & -264.587042 & 259.246 \\
14 & -40.798348 & 0.160 & 40 & -152.333745 & 1094.229 & 65 & -268.594702 & 589.227 \\
15 & -44.806437 & 0.434 & 41 & -156.633479 & 150.091 & 66 & -273.602343 & 348.556 \\
16 & -48.814517 & 2.072 & 42 & -160.641020 & 206.750 & 67 & -278.400953 & 567.831 \\
17 & -52.822588 & 7.775 & 43 & -165.634973 & 418.111 & 68 & -282.683003 & 8649.980 \\
18 & -56.830907 & 19.643 & 44 & -169.642441 & 1377.013 & 69 & -287.462110 & 592.492 \\
19 & -60.812425 & 17.532 & 45 & -174.511633 & 820.333 & 70 & -292.462856 & 353.907 \\
20 & -64.791953 & 22.566 & 46 & -178.519320 & 2858.745 & 71 & -298.405353 & 453.174 \\
21 & -68.783571 & 6.863 & 47 & -183.508227 & 4112.690 & 72 & -302.413229 & 711.709 \\
22 & -72.791747 & 18.985 & 48 & -188.888965 & 119.444 & 73 & -307.421094 & 877.456 \\
23 & -77.302495 & 3.840 & 49 & -192.898412 & 116.635 & 74 & -312.441302 & 1052.447 \\
24 & -81.309508 & 10.076 & 50 & -198.455633 & 1138.199 & 75 & -318.407330 & 1030.447 \\
25 & -85.477376 & 6.137 & 51 & -202.468274 & 281.205 & 76 & -322.414257 & 1157.131 \\
26 & -90.210764 & 34.689 & 52 & -207.480764 & 589.721 & 77 & -327.371999 & 925.977 \\
27 & -94.219798 & 94.305 & 53 & -211.493405 & 634.604 & 78 & -331.379143 & 1540.366 \\
28 & -98.331711 & 9.682 & 54 & -216.636864 & 1062.635 & 79 & -336.798725 & 5205.116 \\
29 & -102.774589 & 223.985 & 55 & -220.646208 & 671.655 & 80 & -340.811371 & 10562.500 \\
30 & -106.835790 & 100.218 & & & & & & \\
\hline \hline
\end{tabular}

TABLE II

Putative global Minima ENERGIES FOR $81 \leq N \leq 146$ With $\rho=14$.

\begin{tabular}{c|r|c|c|r|c|c|c|c}
\hline \hline Atoms & Gen. & Energy & Atoms & Gen. & Energy & Atoms & Gen. & Energy \\
\hline 81 & 270 & -345.823858 & 103 & 15 & -453.307015 & 125 & 1 & -562.448408 \\
82 & 265 & -350.322842 & 104 & 20 & -458.252082 & 126 & 143 & -568.179822 \\
83 & 66 & -354.848991 & 105 & 650 & -462.612300 & 127 & 85 & -572.187270 \\
84 & 137 & -359.276598 & 106 & 148 & -467.316876 & 128 & 40 & -577.237480 \\
85 & 1182 & -363.893075 & 107 & 23 & -472.314113 & 129 & 87 & -582.234940 \\
86 & 4178 & -369.893323 & 108 & 39 & -478.282437 & 130 & 33 & -588.201912 \\
87 & 238 & -373.925239 & 109 & 28 & -482.289824 & 131 & 115 & -593.070343 \\
88 & 128 & -378.918456 & 110 & 5 & -487.305200 & 132 & 120 & -597.259380 \\
89 & 119 & -383.402473 & 111 & 24 & -492.342791 & 133 & 125 & -602.129297 \\
90 & 99 & -388.401652 & 112 & 25 & -498.311001 & 134 & 32 & -607.127628 \\
91 & 75 & -393.973219 & 113 & 17 & -503.220145 & 135 & 20 & -613.089691 \\
92 & 1755 & -397.979930 & 114 & 55 & -507.325288 & 136 & 118 & -617.097211 \\
93 & 51 & -403.000554 & 115 & 57 & -512.284580 & 137 & 57 & -622.141537 \\
94 & 3341 & -408.013642 & 116 & 15 & -517.351072 & 138 & 327 & -627.142993 \\
95 & 153 & -413.288426 & 117 & 38 & -523.244793 & 139 & 17 & -633.108184 \\
96 & 72 & -417.592614 & 118 & 260 & -527.252214 & 140 & 973 & -637.942709 \\
97 & 1039 & -422.799524 & 119 & 32 & -532.291000 & 141 & 199 & -642.118064 \\
98 & 175 & -428.051560 & 120 & 16 & -537.301330 & 142 & 21 & -646.750417 \\
99 & 0 & -433.284404 & 121 & 16 & -543.269060 & 143 & 89 & -652.711658 \\
100 & 40 & -438.323619 & 122 & 256 & -548.157486 & 144 & 3658 & -657.949029 \\
101 & 9 & -444.292784 & 123 & 22 & -552.317919 & 145 & 879 & -662.939679 \\
102 & 36 & -448.299907 & 124 & 313 & -557.235865 & 146 & 7282 & -668.904083 \\
\hline \hline
\end{tabular}




\section{CONCLUSION}

This paper presented the results obtained using a population based search, PBS, for optimising Morse clusters. PBS was able to repeatedly obtain all optimal configurations in the range $5 \leq N \leq 80, \rho=3,6,10,14$ as reported in [2]. In addition, putative global minima have been established for Morse clusters in the range $81 \leq N \leq 146, \rho=14$. The PBS algorithm incorporates and extends key techniques that have been developed in other Morse optimisation algorithms over the last decade. Of particular importance are the use of cut and paste operators, structure niching (using the cluster strain energy as a structure metric), two-phase local search, and a new operator, Directed Optimisation, which extends the previous concept of directed mutation. In addition, PBS is able to operate in a parallel mode for optimising larger clusters.

Future plans for PBS include improving the population creation phase for larger clusters, implementation of additional mutation operators such as stretch and compress and an enhanced version of the Directed Optimisation operator. In addition, with a dedicated 128-node cluster shortly to become available, PBS will be applied to larger Morse clusters and also extended to other, related problems such as Morse Clusters, Mixed Clusters, Benzene Clusters and Water Clusters.

\section{REFERENCES}

[1] M.R. Hoare, Structure and Dynamics of of Simple Microclusters, Advances in Chemical Physics, Vol. 40, pp 49 - 135 (1979).

[2] The Cambridge Cluster Database, http://wwwwales.ch.cam.ac.uk/CCD.html.

[3] Gropp, W.D.; Lusk, E. ANL-96/6 1996 Math Comp Sci Div, Argonne Nat Lab.

[4] M.R. Hoare, J.A. McInnes, "Statistical mechanics and morphology of very small atomic clusters" Faraday Discussions of the Chemistry Society 61 12-24 (1976)

[5] M.R. Hoare, P. Pal, Physical Cluster Mechanics: Statics and Energy Surfaces for Monatomic Systems, Advances in Physics, 20 161-196 (1971).

[6] M.R. Hoare, J.A. McInnes, Morphology and Statistical Statics of Simple Microclusters, Advances in Physics, 32 791-821 (1983).

[7] J.A. Northby, "Structure and binding of Lennard-Jones clusters: $13 \leq$ $N \leq 147$ " Journal of Chemical Physics 87 6166-6178 (1987).

[8] G.L. Xue, "Improvement on the Northby Algorithm for Molecular Confirmation: Better Solutions" Journal of Global Optimisation 4 425440 (1994).

[9] D. Romero, C. Barron, S. Gomez, "The optimal geometry of LennardJones clusters: 148-309" Computer Physics Communications 123 8796 (1999).

[10] Y.H. Xiang, H.Y. Jiang, W.S. Cai, X.G. Shao, "An Efficient Method Based on Lattice Construction and the Genetic Algorithm for Optimisation of Large Kennard-Jones Clusters" Journal of Physical Chemistry A 108 3586-3592 (2004).

[11] Y.H. Xiang, L. Cheng, W. Cai, X. Shao, "Structural Distribution of Lennard-Jones Clusters Containing 562 to 1000 Atoms" Journal of Physical Chemistry A 108 9516-9520 (2004).

[12] D.J. Wales, J.P.K. Doye, "Global Optimisation by Basin-Hopping and the Lowest Energy Structures of lennard-jones Clusters Containing up to 110 Atoms" Journal of Physical Chemistry A 101 5111-5116 (1997).

[13] R.H. Leary, "Global Optimisation on Funneling Landscapes" Journal of Global Optimisation 18 367-383 (2000).

[14] R. H. Leary, J.P.K. Doye, "Tetrahedral global minimum for the 98atom Lennard-Jones cluster" Physical Review E 60 R6320-R6322 (1999).
[15] M. Locatelli, F. Schoen, "Efficient Algorithms for Large Scal Global Optimisation: Lennard-Jones Clusters" Computational Optimisation and Applications 26 173-190 (2003).

[16] D.M. Deaven, N. Tit, J.R. Morris, K. Ho, "Structural Optimization of Lennard-Jones Clusters by a Genetic Algorithm", Chemical Physics Letters 256195 (1996).

[17] B. Hartke, "Global cluster geometry optimization by a phenotype algorithm with Niches: Location of elusive minima, and low-order scaling with cluster size" Journal of Computational Chemistry 201752 (1999).

[18] W.J. Pullan, "An Unbiased Population-Based Search for the Geometry Optimisation of Lennard-Jones Clusters: $2 \leq N \leq 372$ " Journal of Computational Chemistry 26899 (2004).

[19] C. Roberts, R.L. Johnston, N.T. Wilson, "A genetic algorithm for the structural optimisation of Morse clusters" Theoretical Chemistry Accounts 104 123-130 (2000).

[20] J.P.K. Doye, R.H. Leary, M. Locatelli, F. Schoen, "The global optimisation of Morse clusters by potential energy transformations" INFORMS Journal of Computing 16 371-379 (2004).

[21] L. Cheng, J. Yang, "Global minimum Structures of Morse Clusters as a Function of the Range of the Potential: $81 \leq N \leq 160$ " Journal of Physical Chemistry A 111 5287-5293 (2007). 